\title{
Mathematical prediction of the spreading rate of COVID-19 using entropy-based thermodynamic model
}

\author{
A Ghanbari ${ }^{1}$, R Khordad ${ }^{1} *$ and M Ghaderi-Zefrehei ${ }^{2}$ \\ ${ }^{1}$ Department of Physics, College of Science, Yasouj University, Yasouj 75918-74934, Iran \\ ${ }^{2}$ Department of Animal Genetics, Yasouj University, Yasouj 75918-74934, Iran
}

Received: 12 July 2020 / Accepted: 16 September 2020 / Published online: 2 January 2021

\begin{abstract}
In the COVID-19 pandemic era, undoubtedly mathematical modeling helps epidemiological scientists and authorities to take informing decisions about pandemic planning, wise resource allocation, introducing relevant nonpharmaceutical interventions and implementation of social distancing measures. The current coronavirus disease (COVID19) emerged in the end of 2019, Wuhan, China, spreads quickly in the world. In this study, an entropy-based thermodynamic model has been used for predicting and spreading the rate of COVID-19. In our model, all the epidemic details were considered into a single time-dependent parameter. The parameter was analytically determined using four constraints, including the existence of an inflexion point and a maximum value. Our model has been layout-based the Shannon entropy and the maximum rate of entropy production of postulated complex system. The results show that our proposed model fits well with the number of confirmed COVID-19 cases in daily basis. Also, as a matter of fact that Shannon entropy is an intersection of information, probability theory, (non)linear dynamical systems and statistical physics, the proposed model in this study can be further calibrated to fit much better on COVID-19 observational data, using the above formalisms.
\end{abstract}

Keywords: COVID-19; Spreading; Entropy; Thermodynamic

\section{Introduction}

Beginning from late 2019 (December 2019), an outbreak of coronavirus (COVID-19) infection was detected in Wuhan city China [1]. This is the seventh known coronavirus to infect humans and spreads quickly among people [2]. Some forms of coronavirus including severe acute respiratory syndrome (SARS) and the Middle East respiratory syndrome (MERS) were appeared and caused death to many people around the world $[1,3,4]$.

Coronaviruses composed of large RNA viruses that infect humans and animals. In 1966, Tyrell and Bynoe have described coronaviruses, and they have coined the coronaviruses term (Latin: corona $=$ crown) [5]. Four subfamilies of coronaviruses exist, e.g., alpha, beta, gamma and delta. The first and second types originate from mammals, and the third and fourth originate from pigs and birds. Scientists believe that beta-coronavirus can infect humans and may cause severe disease and fatalities [6].

*Corresponding author, E-mail: khordad@yu.ac.ir
It is unanimously thought that the COVID-19 has an animal origin [7-11]. Clinical symptoms of the COVID-19 are fever and cough in addition to other non-specific symptomatology including dyspnea, headache, muscle soreness and fatigue $[12,13]$. The World Health Organization (WHO) has declared a global health emergency on January 30, 2020 [14]. The COVID-19 human-to-human transmission is so fast. This process is like the growth of a tumor or economic growth of a company. Some crucial computational endeavors have been conducted to decipher transmission dynamics of COVID-19 using various mathematical models [15-25]. In this way, Donnelly et al. [15], Riley et al. [16] and Lipsitch et al. [17] have successfully fitted mathematical models to data for SARS.

Recently, some authors have introduced various models to study the COVID-19 in several countries. For example, Wang [26] has considered applications, limitations and potentials of mathematical models for COVID-19. Yang and Wang [27] have proposed a mathematical model to investigate the current outbreak of the coronavirus disease 2019 (COVID-19) in Wuhan, China. Ivorra et al [28] have developed a mathematical model for the spread of the 
coronavirus disease 2019 (COVID-19). Zeb et al [29] have used a mathematical model for coronavirus disease 2019 (COVID-19) containing isolation class.

It is now evident that behavior of COVID-19 epidemic depends on medical interventions, public-service announcements, isolation of people, restriction of individual and social activities. Therefore, it would be very difficult to find all those details of the epidemic by a mathematical model. The dynamics of an epidemic is an interesting and important topic in medicine, physics and biology, and it is usually modeled by differential equations. The proposed models are based on a modified susceptibleexposed-infectious-recovered (SEIR) computational framework.

In this work, we want to determine the number of hospitalized cases (cumulative number of cases minus the number of deaths and the number recovered). Thus, a new approach has been considered to predict this number. In this approach, all the mechanisms controlling the spread are factored into a single parameter. Also, we suppose that the system controlling the spread of COVID-19 epidemic is a thermodynamic system and define an entropy and determine the only parameter by the principle of extreme rate of entropy production. We apply a simple differential equation model with the spread rate forced to satisfy four constraints. Assuming the system for spread to be thermodynamically, the model has been closed by the use of maximum or minimal rate of entropy production.

Computer simulations of cellular automata $[30,31]$ were considered as one of possible methodologies for investigation of epidemiological models [32, 33]. The cellular automata are especially suitable for that purpose because it is usually assumed that agents building the society can be in a discrete number of states. A basic group of epidemiological models originates from the SIR (susceptible-infectious-recovered) model [34] which is, on the one hand, often modified by introduction of additional states of the individual [33, 35]. On the other hand, the interplay between agents and the assumed system topology is another field of possible modification of the considered models [36]. It is frequently assumed that transitions of agents between possible states take place with a constant probability [37, 38]. However, some transitions (like infection) are neighborhood dependent and require that a given agent is in contact with at least one infectious agent [39]. If a susceptible agent is in contact with a larger number of infectious individuals, it can become infectious with a higher probability. This originates not from a larger probability of infection in a single interaction but from a larger number of trials. Accordingly, we can adopt the entropic-based model to fit data from COVID-19 and predict its dynamics.
Entropy is one of the significant quantities in statistical mechanics. This quantity is applicable in studying biological systems [40, 41]. Entropy shows missing information on the state of a system, and it is measure disorder of a system. Here, this quantity has been used to predict the behavior of our system. In this way, in Sect. 2, we have presented the entropic-based mathematical model and defined some parameters. Application, validation and discussion of the model in some countries COVID-19 data are explained in Sect. 3. Finally, the conclusion of our proposed model efficiency and generality is presented in Sect. 4.

\section{Mathematical framework}

\subsection{The entropy-based thermodynamic basic model}

First, we consider $f(t)$ function to be the number of confirmed daily cases, as the cumulative cases number. This function is subtracted from the daily deaths and recovery cases, since death and recovery cases are also parts of players in the thermodynamic system. In this research, we have considered the epidemic as a thermodynamic system and followed the approach for the rate of vibration excitation and chemical reaction $[42,43]$. Thus, we have supposed that the rate of increase (decrease) would be proportional to the cumulative cases at the previous day:

$\frac{\mathrm{d} f(t)}{\mathrm{d} t}=\alpha(t) f(t)$,

where $\alpha(t)$ denotes a time-dependent parameter. The determination of this parameter depends on the spreading and controlling of the epidemic. Here, we have not considered the dependence of the parameter on other quantities e.g., population.

To proceeding along, here, we impose four constraints on this parameter:

(i) The parameter $\alpha(t)$ must have the dimension of $t^{-1}$. We know that Eq. (1) is the master equation, and $\alpha(t)$ shows the transition probabilities for various pairs of states. The parameter is proportional to inverse time. Therefore, we have selected the simplest choice as $\alpha(t) \sim t^{-1}$. With this selection, the following constraint is satisfied $(t \rightarrow 0, \alpha \rightarrow \infty)$.

(ii) There is an exponential enhancement of smooth spread to start at the initial stage, $\alpha(0) \rightarrow \infty$.

(iii) On a given day, $t=L$, the rate must decrease. Mathematically, it means that $\frac{\mathrm{d}^{2} f(t)}{\mathrm{d} t^{2}}$ vanishes at $t=L$; this is called inflexion date. Substituting Eq. (1) into $\frac{\mathrm{d}^{2} f(t)}{\mathrm{d} t^{2}}=0$ yields 
$\frac{\mathrm{d} \alpha}{\mathrm{d} t}+\alpha^{2}=0, \quad t=L$

(iv) $f(t)$ has a maximum; this means that for $t=D$, $\alpha(D)=0$.

According to above imposed constraints, it is supposed that $\alpha(t)$ to be an analytical function of time. The following function would satisfy the four constraints, and it is sufficiently efficient

$\alpha(t)=-\frac{b}{t} \ln \left(\frac{t}{D}\right)$

where $b=\frac{1-\ln (L / D)}{\ln ^{2}(L / D)}$. It should note that above function is not unique, and we can select other functions which satisfy the constraints. To find the function of $f(t)$ analytically, we have chosen $\alpha(t)$ as Eq. (3) that is sufficiently simple. Inserting Eq. (3) into Eq. (1), we obtain the following relation:

$f(t)=\frac{k}{\sqrt{2 \pi} \sigma t} \exp \left(-\frac{(\ln (t)-\mu)^{2}}{2 \sigma^{2}}\right)$

Here $\sigma$ and $k$ are proportion constants and $\mu=\ln D+\sigma^{2}$. It is noteworthy that $\sigma$ can be computed by of the principle of the extreme rate of entropy production.

\subsection{Extreme rate of entropy production}

The principle of the extreme rate of entropy production can be employed as a reliable tools to calculate model parameters [44, 45]. Santos et al. [46] have shown that the entropy production of a quantum system undergoing opensystem dynamics can be formally split up into a term that only depends on population unbalances. Tsuruyama [47] has considered the locality of the second law of thermodynamics and showed that entropy can be divided into entropy derived from a chemical reaction and entropy produced by the diffusion of signaling molecule. It is to be noted that the width of the curve $f(t) \propto t$ can be characterized by $\sigma$. The wider the curve is, the larger is the (Shannon) entropy. The intrinsic spread mechanism of virus and the large mixing activity of the population tend to make the curve wider.

As the maximum dissipation rate is obtained, the width would cease to enhance. Therefore, maximum dissipation rate corresponds to the extreme rate of entropy production. It is related to

$\frac{\mathrm{d}^{2} S(\sigma, \eta)}{\mathrm{d} \sigma^{2}}=0$,

where $S(\sigma, \eta)$ is the Shannon entropy and it can be written as
$S(\sigma, \eta)=-\int_{0}^{\infty} F(t) \ln F(t) \mathrm{d} t^{\eta}$

Here, $F(t)=t^{1-\eta} f(t)$ with $\eta=3$. This value is in the usual entropy definition [44].

The solution of the integral is

$S(\sigma, \eta)=\eta\left(\ln (\sqrt{2 \pi} \sigma)+\eta\left(\ln D+\sigma^{2}\right)+\frac{1}{2}\right)$

Equation (5) holds if and only if

$\sigma=\frac{1}{\sqrt{2 \eta}} \approx 0.0408$, for $\eta=3$

With the value of $\sigma$, the intrinsic spread mechanism is balanced by the dissipation mechanism. To obtain the relationship between two typical dates $D$ (maximum number of confirmed daily cases) and $L$ (inflexion date), we should employ the definion of inflexion point. The inflexion point is the date at which the multiple controlling measure takes effect.

We inserted Eq. (4) into $\left.\frac{\mathrm{d}^{2} f(t)}{\mathrm{d} t^{2}}\right|_{t=L}=0$; then we have

$D=L \exp \left(\frac{1}{2} \sigma^{2}+\frac{1}{2} \sqrt{4 \sigma^{2}+\sigma^{4}}\right)$

Applying Eq. (4), one can determine a relation between $f(D)$ and $f(L)$ as

$f(D)=F(L) \exp \left(-\sigma^{2}-\frac{1}{2} \sqrt{4 \sigma^{2}+\sigma^{4}}+\frac{1}{2}\left(\frac{3}{2} \sigma+\frac{1}{2} \sqrt{4+\sigma^{2}}\right)^{2}\right)$

According to Eq. (8), the following important relations can be grasped

$\left.\frac{D}{L}\right|_{\eta=3}=1.649,\left.\quad \frac{f(D)}{f(L)}\right|_{\eta=3}=2.120$

By applying the above relations, we can predict the maximal number of confirmed daily cases and the day in which this maximum appears. The maximum number of infected individuals and the time at which this maximum occurs can be related to the number and time corresponding to the critical date.

First, we compute the following relation

$\left.\frac{\mathrm{d} f(t)}{\mathrm{d} t}\right|_{t=L}=-\frac{1}{\sigma^{2} L} f(L) \ln \frac{L}{D}$

which yields

$L=\left(\frac{1}{2}+\frac{1}{2} \sqrt{\frac{4}{\sigma^{2}}+1}\right) \frac{f(L)}{\left.\frac{\mathrm{d} f(t)}{\mathrm{d} t}\right|_{t=L}}=\frac{3 f(L)}{\left.\frac{\mathrm{d} f(t)}{\mathrm{d} t}\right|_{t=L}}$

We want to know the initial date for which the spreading 
Table 1 Confirmed daily cases and daily deaths of COVID-19 Worldwide, China, and Iran from January 23, 2020, to April 5, 2020 (Data are given from https://www.worldometers.info/coronavirus/)

\begin{tabular}{|c|c|c|c|c|c|c|}
\hline \multirow[t]{2}{*}{ The date } & \multicolumn{3}{|c|}{ Confirmed daily cases } & \multicolumn{3}{|l|}{ Daily deaths } \\
\hline & Worldwide & China & Iran & Worldwide & China & Iran \\
\hline Jan 23 & 398 & 259 & - & - & 8 & - \\
\hline Jan 24 & 472 & 457 & - & - & 16 & - \\
\hline Jan 25 & 698 & 688 & - & - & 15 & - \\
\hline Jan 26 & 785 & 769 & - & 24 & 24 & - \\
\hline Jan 27 & 1781 & 1771 & - & 26 & 26 & - \\
\hline Jan 28 & 1477 & 1459 & - & 26 & 26 & - \\
\hline Jan 29 & 1755 & 1737 & - & 38 & 38 & - \\
\hline Jan 30 & 2010 & 1981 & - & 43 & 43 & - \\
\hline Jan 31 & 2127 & 2099 & - & 46 & 46 & - \\
\hline Feb 1 & 2603 & 2589 & - & 45 & 45 & - \\
\hline Feb 2 & 2833 & 2825 & - & 55 & 57 & - \\
\hline Feb 3 & 3239 & 3235 & - & 65 & 64 & - \\
\hline Feb 4 & 3915 & 3884 & - & 66 & 65 & - \\
\hline Feb 5 & 3721 & 3694 & - & 73 & 73 & - \\
\hline Feb 6 & 3173 & 3143 & - & 73 & 73 & - \\
\hline Feb 7 & 3437 & 3385 & - & 86 & 86 & - \\
\hline Feb 8 & 2676 & 2652 & - & 89 & 89 & - \\
\hline Feb 9 & 3001 & 2973 & - & 97 & 97 & - \\
\hline Feb 10 & 2546 & 2467 & - & 108 & 108 & - \\
\hline Feb 11 & 2035 & 2015 & - & 97 & 97 & - \\
\hline Feb 12 & 4153 & 4108 & - & 146 & 146 & - \\
\hline Feb 13 & 5151 & 5090 & - & 122 & 121 & - \\
\hline Feb 14 & 2662 & 2641 & - & 143 & 143 & - \\
\hline Feb 15 & 2097 & 2008 & - & 143 & 142 & - \\
\hline Feb 16 & 2132 & 2048 & - & 106 & 105 & - \\
\hline Feb 17 & 2003 & 1888 & - & 98 & 98 & - \\
\hline Feb 18 & 1852 & 1749 & - & 136 & 136 & - \\
\hline Feb 19 & 516 & 391 & - & 117 & 114 & - \\
\hline Feb 20 & 977 & 889 & - & 121 & 118 & - \\
\hline Feb 21 & 996 & 823 & 13 & 131 & 109 & 2 \\
\hline Feb 22 & 978 & 648 & 11 & 100 & 97 & 2 \\
\hline Feb 23 & 554 & 214 & 14 & 158 & 150 & 2 \\
\hline Feb 24 & 882 & 508 & 18 & 81 & 71 & 4 \\
\hline Feb 25 & 741 & 406 & 34 & 64 & 52 & 4 \\
\hline Feb 26 & 992 & 433 & 44 & 37 & 29 & 3 \\
\hline Feb 27 & 1292 & 327 & 106 & 57 & 44 & 7 \\
\hline Feb 28 & 1503 & 427 & 143 & 65 & 47 & 8 \\
\hline Feb 29 & 1989 & 573 & 205 & 54 & 35 & 9 \\
\hline Mar 1 & 1981 & 202 & 385 & 73 & 42 & 11 \\
\hline Mar 2 & 1858 & 125 & 523 & 67 & 31 & 12 \\
\hline Mar 3 & 2573 & 119 & 835 & 85 & 38 & 11 \\
\hline Mar 4 & 2298 & 139 & 586 & 83 & 31 & 15 \\
\hline Mar 5 & 3111 & 143 & 591 & 102 & 30 & 16 \\
\hline Mar 6 & 3625 & 99 & 1234 & 107 & 28 & 16 \\
\hline Mar 7 & 4049 & 44 & 1076 & 105 & 27 & 21 \\
\hline Mar 8 & 3892 & 40 & 743 & 228 & 22 & 49 \\
\hline
\end{tabular}

Table 1 continued

\begin{tabular}{|c|c|c|c|c|c|c|}
\hline \multirow[t]{2}{*}{ The date } & \multicolumn{3}{|c|}{ Confirmed daily cases } & \multicolumn{3}{|l|}{ Daily deaths } \\
\hline & Worldwide & China & Iran & Worldwide & China & Iran \\
\hline Mar 9 & 4390 & - & 595 & 198 & 17 & 43 \\
\hline Mar 10 & 4567 & - & 881 & 271 & 22 & 54 \\
\hline Mar 11 & 7266 & - & 958 & 332 & 11 & 63 \\
\hline Mar 12 & 8295 & - & 1075 & 353 & 7 & 75 \\
\hline Mar 13 & 10907 & - & 1289 & 447 & 13 & 85 \\
\hline Mar 14 & 11059 & - & 1365 & 405 & 10 & 97 \\
\hline Mar 15 & 13042 & - & 1209 & 687 & 14 & 113 \\
\hline Mar 16 & 12897 & - & 1053 & 642 & 13 & 129 \\
\hline Mar 17 & 15745 & - & 1178 & 817 & 11 & 135 \\
\hline Mar 18 & 20585 & - & 1192 & 972 & 8 & 147 \\
\hline Mar 19 & 26158 & 39 & 1046 & 1079 & 3 & 149 \\
\hline Mar 20 & 30648 & 41 & 1237 & 1356 & 7 & 149 \\
\hline Mar 21 & 29429 & 46 & 966 & 1625 & 6 & 123 \\
\hline Mar 22 & 32480 & 39 & 1028 & 1629 & 9 & 129 \\
\hline Mar 23 & 41371 & 78 & 1411 & 1873 & 7 & 127 \\
\hline Mar 24 & 43744 & 47 & 1762 & 2381 & 4 & 122 \\
\hline Mar 25 & 48461 & 67 & 2206 & 2388 & 6 & 143 \\
\hline Mar 26 & 60830 & 55 & 2389 & 2791 & 5 & 157 \\
\hline Mar 27 & 64501 & 54 & 2926 & 3270 & 3 & 144 \\
\hline Mar 28 & 66761 & 45 & 3076 & 3518 & 5 & 139 \\
\hline Mar 29 & 60263 & 52 & 2901 & 3204 & 5 & 123 \\
\hline Mar 30 & 61351 & 79 & 3186 & 3709 & 7 & 117 \\
\hline Mar 31 & 73620 & 36 & 3110 & 4535 & 6 & 141 \\
\hline Apr 1 & 76871 & - & 2988 & 4889 & 4 & 138 \\
\hline Apr 2 & 79864 & - & 2875 & 5979 & 4 & 124 \\
\hline Apr 3 & 101566 & - & 2715 & 5714 & 3 & 134 \\
\hline Apr 4 & 84821 & - & 2560 & 5800 & 4 & 158 \\
\hline
\end{tabular}

of the epidemic triggers. For this purpose, we must determine the value $L$. This value has been obtained in Eq. (13).

\section{Validation and discussion of the proposed model}

Before using the model on real data, we must determine some model parameters. Firstly, we characterized the number $F=f(L)$ at the inflexion date (the date that $\frac{\mathrm{d} f(t)}{\mathrm{d} t}$ tends to reduce) and $\left.\frac{\mathrm{d} f(t)}{\mathrm{d} t}\right|_{t=L}$ using the reported daily cases data. The constant $k$ has been obtained by $f(L)=F$ in Eq. (4). Finally, we used Eq. (13) to find $L$. Afterward, using Eq. (11), we predicted $D$ and $f(D)$ and plot the curve $f(t) \sim t$ to predicted the number $f(t)$ for $L<t$. 

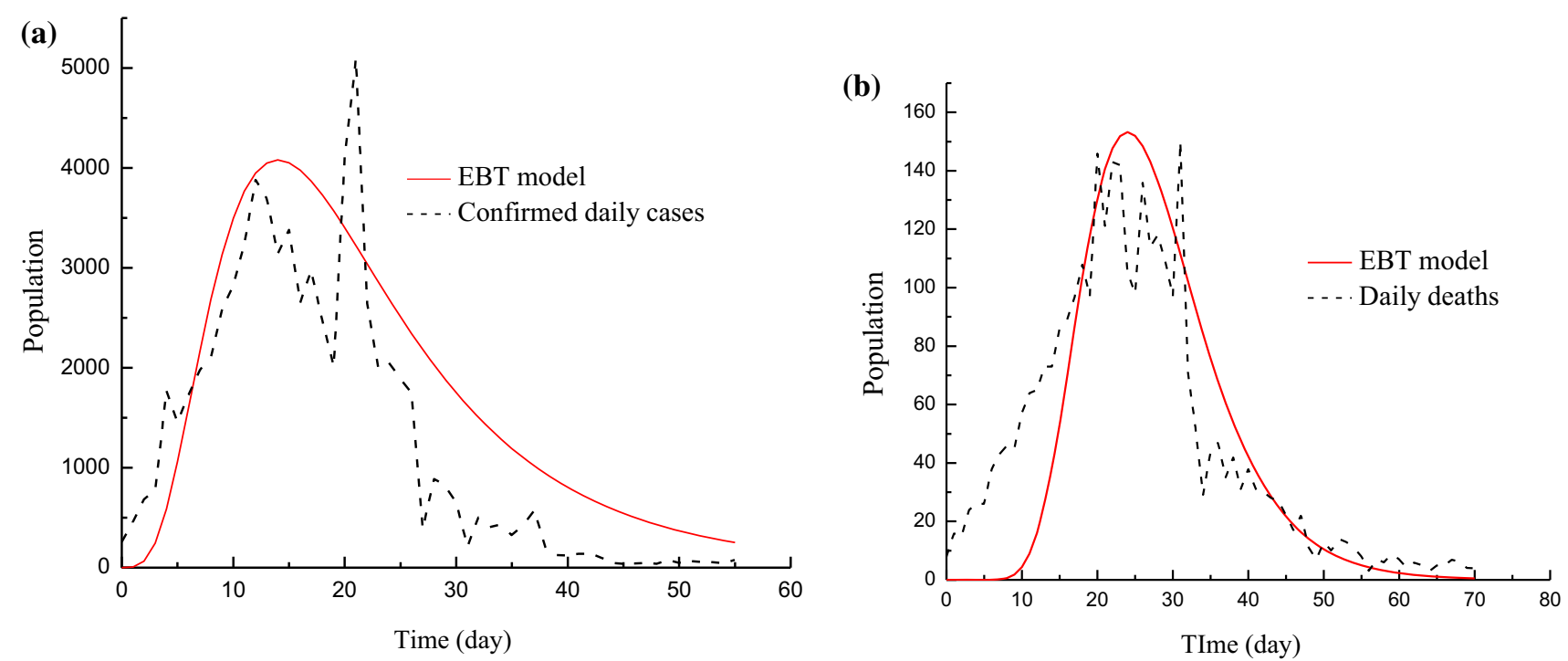

Fig. 1 Comparison of the results of China COVID-19 with entropy-based thermodynamic model: (a) confirmed daily cases and (b) daily deaths
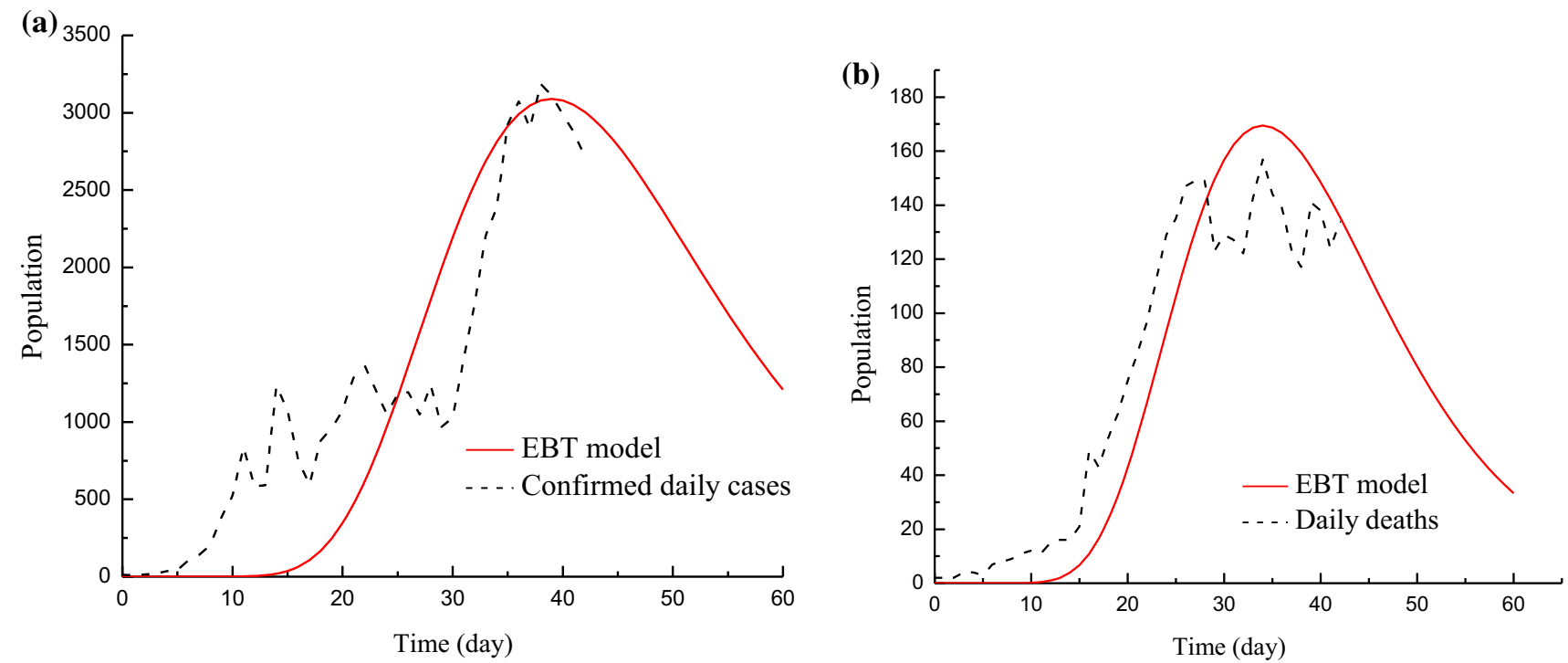

Fig. 2 Comparison of the results of Iran COVID-19 with entropy-based thermodynamic model: (a) confirmed daily cases and (b) daily deaths

Then, we find the inflexion point as follows. We record the reported the number $f(t)$ for each day and plotted the curve $g(t)=f(t)-f(t-1)$. When we observed that $g(t)$ reaches a peak (denoted as $G$ ) at $t=L$, then $L$ is considered as the inflexion point.

Also $L$ is calculated numerically as

$L=\frac{3 \bar{F}}{G}$

Here, $\bar{F}=(2 F-G) / 2$ is the number of $f$ averaged daily cases over two consecutive dates.

After determining the model parameters, we test the model for China and Iran confirmed COVID-19 data, since as it can be seen, only these two countries have a peak in data reported. Table 1 shows the reported data from January 23, 2020, to April 5, 2020. In Fig. 1(a) and (b), we have compared our analytical model with confirmed daily cases and daily deaths of China, respectively. As can be seen, there is fairly good agreement between our model and reported data. At initial parts of the curve, our model has a good agreement with reported data. Then, nearly peaks of curves this agreement gets biased as time passes, and this agreement gets disappeared. Figure 2(a) and (b) shows comparison of the model with confirmed daily cases and daily deaths of Iran, respectively. The figures show there is a rather good agreement between data and proposed model. In this case, we don't have enough data so we just can predict the future of cases in Iran. Figure 3(a) and 

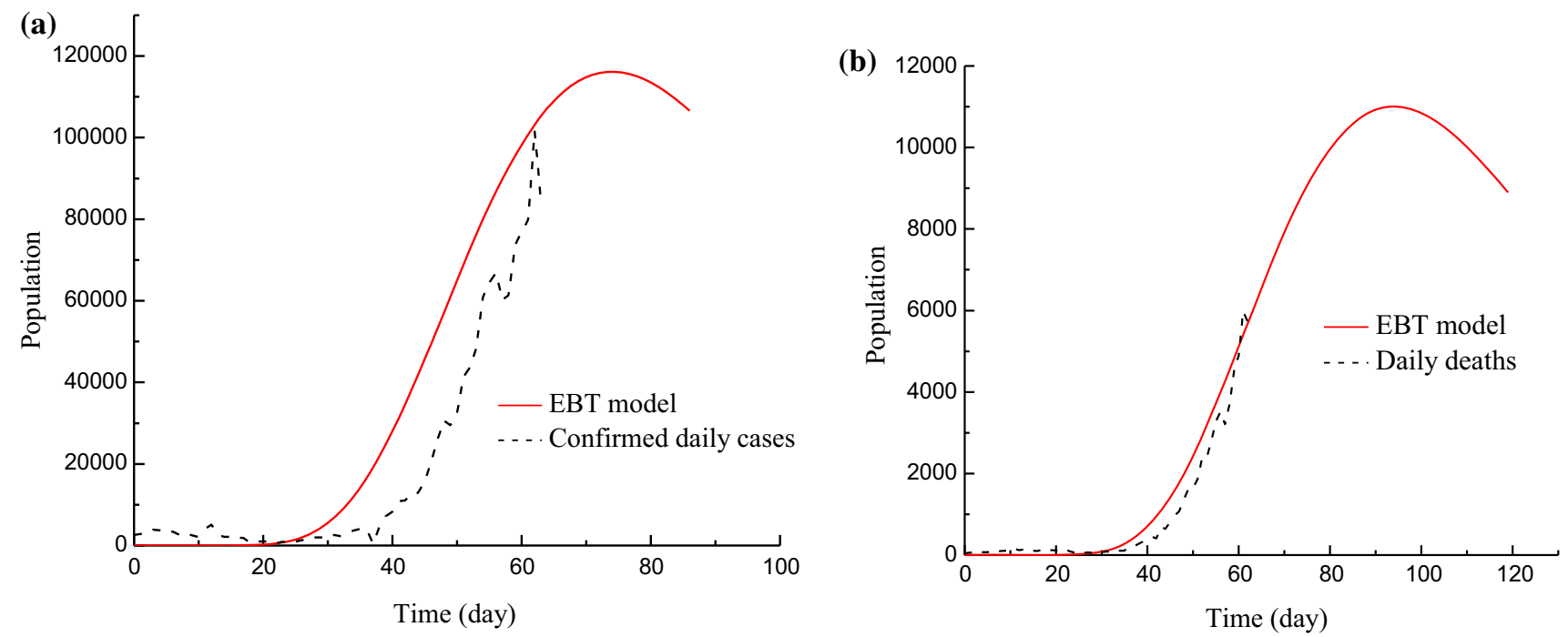

Fig. 3 Comparison of the results of worldwide COVID-19 with entropy-based thermodynamic model: (a) confirmed daily cases and (b) daily deaths

(b) shows confirmed daily cases and daily deaths of worldwide. In this case, no sign of curve peak could be observed in reported data. According to the model, we can predict the behavior of worldwide diagram and find behavior dynamics for it. Finally, we can predict the behavior of cases for other countries and regions. The proposed mathematical perspective gives a central position to entropy and relative entropy in tracking down the dynamic behavior of COVID-19 and provides insights into the notions of randomness, typicality and disorder in COVID-19. However, relevance of in life science and COVID-19 modeling is yet to be demonstrated and worked out.

\section{Conclusions}

In this work, we have studied the entropic model of coronavirus (COVID-19). To reach the goal, we have used the entropy-based thermodynamic principle and have introduced a mathematical model that almost fitted well to the reported data. Accordingly, the proposed model can be used to predict the future of COVID-19 propagation. It should note that the simplicity of the model is an advantage point. The details of epidemic have been ignored in our model. We have used the model for the first peak in the infection. More comparison needs to be done to further assess the validity. This can be considered in future studies.

It is needless to say that many factors play pivotal roles in spreading and propagating of COVID-19 in the community. The proposed model layouts a template for those factor to be well calibrated in it, given accessibility to enough amount of data and having semantic conceptual relations among them.

\section{References}

[1] W H Organization WHO Statement Regarding Cluster of Pneumonia Cases in Wuhan, China (Beijing: WHO) 912 (2020)

[2] N Zhu, et al. New England Journal of Medicine 385543 (2020)

[3] C W Lam, M H Chan and C K Wong Clinical Biochemist Reviews 25121 (2004)

[4] E I Azhar, et al. Infectious Disease Clinics 33891 (2019)

[5] D Tyrrell and M Bynoe Lancet 1276 (1966)

[6] W Tan, et al. China CDC Weekly 261 (2020)

[7] J F W Chan, et al. Lancet 395514 (2020)

[8] C Huang, et al. Lancet 395497 (2020)

[9] L T Phan, et al. New England Journal of Medicine 382872 (2020)

[10] Q Li, et al. New England Journal of Medicine 382123 (2020)

[11] S Chatterjee, et al. Indian. J. Phys. (2020) https://doi.org/10.1007/s12648-020-01766-8

[12] C P E R E Novel Zhonghua liuxingbingxue zazhi 41145 (2020)

[13] C Wang, et al. Lancet 395470 (2020)

[14] E Mahase Bmj 368 m408 (2020)

[15] C A Donnelly, et al. Lancet 3611761 (2003)

[16] S Riley, et al. Science 3001961 (2003)

[17] M Lipsitch, et al. Science 3001966 (2003)

[18] C Dye and N Gay Science 3001884 (2003)

[19] N Jia and L Tsui North American Actuarial Journal 928 (2005)

[20] D J Watts, et al. Proceedings of the National Academy of Sciences 10211157 (2005)

[21] E Kenah and J M. Robins Physical Review E 76036113 (2007)

[22] L Bian and D Liebner Transactions in GIS 11155 (2007)

[23] X Yu, et al. Physica A 3871421 (2008)

[24] B Dybiec The European Physical Journal B 67377 (2009)

[25] B Dybiec, A Kleczkowski and C A Gilligan Journal of the Royal Society Interface 6941 (2009)

[26] J Wang J. Public. Healtb. Emerg. 49 (2020)

[27] C Yang and J Wang Math. Biosci. Engin. 172708 (2020) 
[28] B Ivorra et al Commun. Nonlinear Sci. Numer. Simulat. 88 105303 (2020)

[29] A Zeb et al Biomed Res. Int. 73452402 (2020)

[30] S Wolfram Rev. Mod. Phys. 55601 (1983)

[31] S Wolfram A New Kind of Science (Champaign: Wolfram Media) (2002)

[32] C Rhodes and R M Anderson Theor. Popul. Biol. 52101 (1997)

[33] B Dybiec, A Kleczkowski and C Gilligan Phys. Rev. E 70 066145 (2004)

[34] R M Anderson, B Anderson and R M May Infectious Diseases of Humans: Dynamics and Control (Oxford University Press) 1992.

[35] E Ahmed and H Agiza Physica A 253347 (1998)

[36] N Boccara and K Cheong J. Phys. A 263707 (1993)

[37] B Dybiec, A Kleczkowski and C Gilligan Acta Phys. Polo. B 36 12 (2005)

[38] A Kleczkowski, B Dybiec and C Gilligan, arXiv preprint physics/0608141 (2006)

[39] P Grassberger Math. Biosci. 63157 (1983)
[40] R Khordad and H R Sedehi The European Physical Journal Plus 130246 (2015)

[41] H Husi Feature Selection in Microarray Data Using Entropy Information-Computational Biology (2019)

[42] Z N Wu Probabil. Eng. Mech. 18241 (2003)

[43] J D Anderson Jr Hypersonic and high Temperature Gas Dynamics, 2nd edn. (Reston: American Institute of Aeronautics and Astronautics, Inc.) (2006)

[44] H Ziegler An Introduction to Thermomechanics (Amsterdam: NorthHolland Publisher) (1983)

[45] W B Wang Z N Wu, C F Wang and R F Hu Sci. Chin. Phys. Mech. Astron. 562143 (2013)

[46] J P Santos, et al. npj Quant. Inform. 51 (2019)

[47] T Tsuruyama J. Theor. Biol. 47284 (2019)

Publisher's Note Springer Nature remains neutral with regard to jurisdictional claims in published maps and institutional affiliations. 